\title{
Applying the principle of orthogonality of high dimensional random vectors to obtain high-density, large-volume 3D holographic display
}

\author{
Ghaith Makey ${ }^{1}$, Özgün Yavuz ${ }^{2}$, Denizhan Koray Kesim ${ }^{2}$, Ahmet Turnalı ${ }^{2}$, Parviz Elahi ${ }^{1}$, Johnny Toumi ${ }^{3}$, \\ Moustafa Sayem El-Daher ${ }^{3}$, Serim Ilday ${ }^{4}$, Onur Tokel ${ }^{1,4}$, F. Ömer Ilday ${ }^{1,2,4, \uparrow}$ \\ ${ }^{I}$ Department of Physics, Bilkent University, Ankara, 06800, Turkey \\ ${ }^{2}$ Department of Electrical and Electronics Engineering, Bilkent University, Ankara, 06800, Turkey \\ ${ }^{3}$ Higher Institute for Laser Research and Applications, Damascus University, Damascus, Syria \\ ${ }^{4}$ UNAM - National Nanotechnology Research Center and Institute of Materials Science and Nanotechnology, Bilkent \\ University, Ankara, 06800, Turkey \\ Authore-mail address: ilday@bilkent.edu.tr
}

\begin{abstract}
The efforts toward truly 3D displays are far from exploiting the full potential of holography. Here, we apply the principle of orthogonality of high dimensional random vectors to obtain unprecedented dense, large volume holograms.

OCIS codes: (090.1760) Computer Holography; (090.2870) Holographic Display; (230.6120) Spatial Light Modulators
\end{abstract}

Computer generated holograms (CGHs) are highly optimizable tools tailored to control phase and intensity of light over space. The diffraction phenomenon is the prime player that makes CGHs work, described mathematically by Fraunhofer diffraction for high resolution 2D projection (Fourier holograms), and Fresnel diffraction for deep 3D projection (Fresnel holograms). Such control can be directed toward 3D display technology, also known as the holy grail of holography. There have been serious efforts toward improving the 3D holographic control of light, yet such efforts are limited in terms of the number of the projected planes, the depth of field, or the resolution of the total projection [1-2].

Here, we break all these limitations simultaneously with two processes: First we combine Fourier and Fresnel holography by the utilization of stacked Fresnel zone plates (FZP). FZPs are added to manipulate the propagation kernel of Fresnel diffraction locally to be flat at specific depths away from the hologram, thus reducing Fresnel diffraction to Fraunhofer diffraction at image planes. That allows the usage of Fourier holography techniques to generate multiplane projections that can be positioned along the optical axis at arbitrary distances decided by the FZPs, the multiplane projection can be assembled to build 3D objects such as the 100-planes Pantheon of Rome (Fig.1A). Second, we use the property of orthogonality of asymptotically large-dimensional random vectors to reduce the crosstalk between the image planes. That is done by inserting pure phase diffusers at each image plane, rendering the images mutually nearly random and reducing the crosstalk from one to other (Fig.1B). Each image plane can be regarded as an $n$-dimensional vector in complex space, where $n$ is the number of pixels of the hologram, typically a large number. Then, the elimination of crosstalk between the image planes is equivalent to demanding these $n$-vectors to be orthogonal, and here comes the well-known fact that random vectors become asymptotically orthogonal in the limit of $n$ at infinity (Fig.1C). Such approach is crucial to project back to back images to build up 3D projections with unpreceded density and volume.

We demonstrate 3D Fresnel holograms that project high resolution, multiplane, back-to-back images forming 3D projection with reduced crosstalk. Our demonstrations include the projection of 100 plane of Pantheon (Fig.1A), and 1000-planes projection of a rotating rectangle, which advances the state-of-art by two orders of magnitude in terms of number of planes (Fig.1D). We further showcase our holograms capability in experiment by applying our holograms on liquid crystal on silicon spatial light modulator (LCoS SLM). We show the optical reconstruction of unprecedented projection of 11 well-separated back to back images forming the letters of BILKENT UNIV (Fig.1E). The holograms are universally applicable to all types of static and dynamic phase holographic media, allowing enhancing the technology of 3D projection to such state-of-art media like in metamaterials [3], and in-silicon holograms [4]. 
A

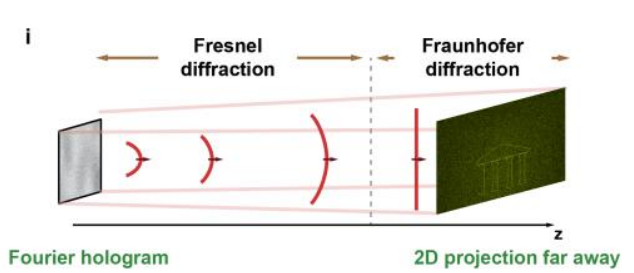

ii

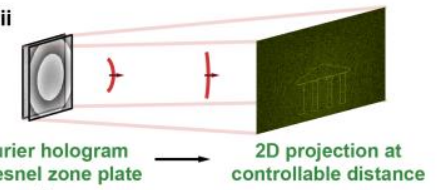

\& Fresnel zone plate controllable distance

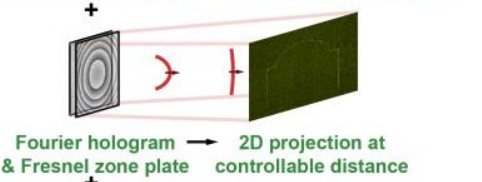

\& Fresnel zone plate controllable distance

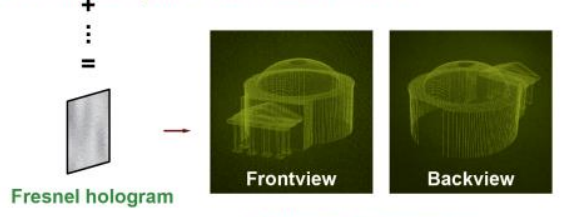

Multiplane 3D projection

E
B

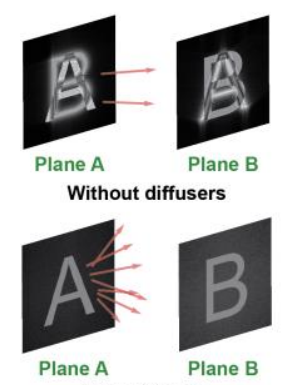

With diffusers
C

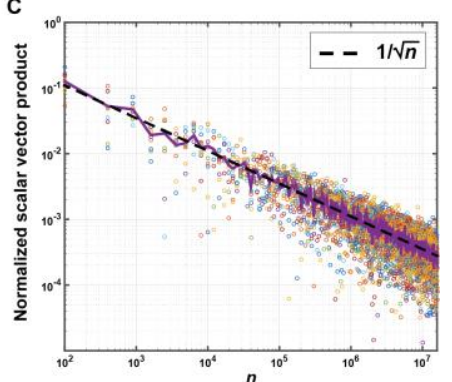

D

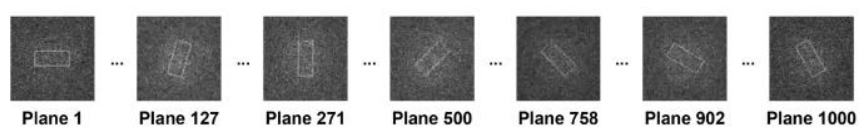

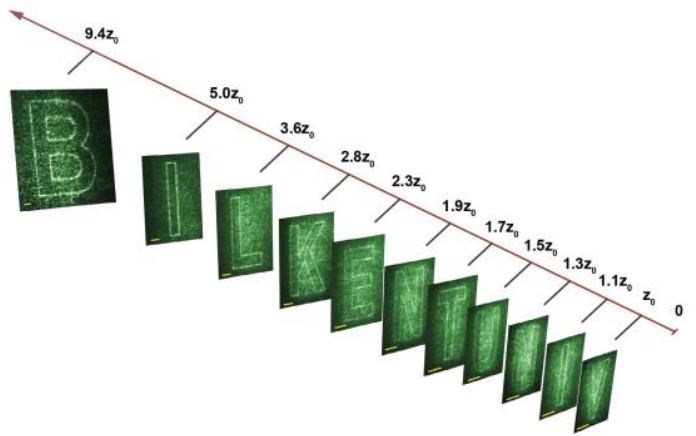

Fig. 1. (A) The concept and implementation of 3D Fresnel holograms. i. Fourier holograms are designed to work at Fraunhofer diffraction condition, projecting 2D image away from the hologram. ii. FZP can be added to shift the image of Fourier hologram along the optical axis, and

by repeating the operation for multiple images and adding the resulted holograms together in the complex form we obtain a single Fresnel hologram that is able to project full 3D projection. The 3D projection here is compiled from simulation result to form the Pantheon of Rome, composed of 100 planes. (B) The effect of adding diffuser to each source image. (i) When no diffuser is used, unwanted crosstalk will form between plane A and B. In contrast, when diffuser is added to the projected image on plane A, the image will be "diffused" away from the focal plane of the corresponding FZP, significantly reducing the crosstalk. (C) The orthogonality of high dimensional random vectors. We calculate the normalized scalar vector product (NSVP) between two images to be projected of a hologram after adding random phase to each, with the size of these images $(n)$ varying from $10 \times 10$ to $4000 \times 4000$ pixels. The value of NSVP is decreasing dramatically as the size of the images increases, indicating that the orthogonality between the two images is increasing at the same rate. (D) The simulation results of a large volume high-

density 3D Fresnel hologram. The hologram is extending $50 \mathrm{~cm}$ in depth and the 1000 on-axis images are simulated using a $4000 \times 4000$ hologram. The image order is given under the chosen images of the projection. (E) Optical reconstruction results. Eleven-plane projection of letters spelling BILKENT UNIV. The distances from the hologram are, 170, 91, 65, 51, 42, 35, 31, 27, 24, 20 and $18 \mathrm{~cm}$. Scale bars: $2 \mathrm{~mm}$. The hologram size is $512 \times 512$ pixels, and applied on LCoS SLM with pixel size $20 \mu \mathrm{m}$.

We show that the quality and number of planes scale up with the number of pixels available in the holographic media, which is expanding rapidly with the current trend in display technologies. Our algorithm can be used for realtime update-rate dynamic projection where we were able to generate the full projection of (Fig.1E) in $80 \mathrm{~ms}$ by implementing GPU computing in CGH code. Our method is tailored mainly toward holographic 3D display applications, and can also be applied to volumetric displays technologies to enable multi-foci control of the tweezing (or plasma inducing) beam [5]. Further, we expect the method to enhance the projection capabilities of holograms in multiple fields including the integrated holograms in modern electro-optical devices [6].

\section{References}

[1] K. Wakunami et al., "Projection-type see-through holographic three-dimensional display,” Nat Commun 7, (2016).

[2] H. Yu, K. Lee, J. Park, Y. Park, “Ultrahigh-definition dynamic 3D holographic display by active control of volume speckle fields,” Nat. Photonics 11, 186-192 (2017).

[3] S. C. Malek, H.-S. Ee, R. Agarwal, "Strain multiplexed metasurface holograms on a stretchable substrate," Nano Lett. 17, 3641 (2017).

[4] O. Tokel et al., "In-chip microstructures and photonic devices fabricated by nonlinear laser lithography deep inside silicon," Nat. Photonics 11, 639-645 (2017).

[5] D. E. Smalley et al., “A photophoretic-trap volumetric display,” Nature 553, 486-490 (2018).

[6] Z. Yue, G. Xue, J. Liu, Y. Wang, M. Gu, “Nanometric holograms based on a topological insulator material,” Nat Commun 8, 15354 (2017). 